\title{
PRODUTIVIDADE DO MILHO RELACIONADA COM A RESISTÊNCIA MECÂNICA À PENETRAÇÃO DO SOLO SOB PREPARO CONVENCIONAL
}

\author{
ONÃ S. FREDDI ${ }^{1}$, MOREL P. CARVALHO², VALDENIR VERONESI JÚNIOR ${ }^{3}$, \\ GUILHERME J. CARVALHO
}

\begin{abstract}
RESUMO: A resistência mecânica do solo à penetração exerce grande influência sobre o desenvolvimento vegetal, uma vez que o crescimento das raízes e a produtividade das culturas variam de forma inversamente proporcional ao seu valor. O objetivo do trabalho foi estudar a variabilidade espacial e a correlação entre a produtividade do milho (PG) e a resistência mecânica do solo à penetração (RP) de um Latossolo Vermelho sob preparo convencional, em área pertencente à Fazenda Experimental de Ensino e Pesquisa da Faculdade de Engenharia - FE/UNESP - Câmpus de Ilha Solteira. Foram definidos 133 pontos de amostragem no campo, segundo malha com espaçamento regular de 10,0 m, nos quais foram medidas a RP, a umidade gravimétrica (UG), nas camadas de 00,$10 ; 0,10-0,20 ; 0,20-0,30$ e 0,30-0,40 m, e a PG. O modelo exponencial mostrou-se adequado para descrever todos os semivariogramas experimentais construídos. A RP e a UG apresentaram moderada dependência espacial, com alcance variando entre 20,2 e 63,0 m. A PG apresentou forte dependência espacial com alcance de $15,3 \mathrm{~m}$. A RP não apresentou correlação espacial com a PG. Valores de RP variando entre 0,9 e 2,0 MPa não restringiram a PG.
\end{abstract}

PALAVRAS-CHAVE: geoestatística, krigagem, Zea mays.

\section{RELATIONSHIP BETWEEN MAIZE YIELD AND SOIL MECHANICAL RESISTANCE TO PENETRATION UNDER CONVENTIONAL TILLAGE}

\begin{abstract}
The soil mechanical resistance to penetration shows high influence on vegetal development since the growth of the roots and the crops productivity change in the inversely proportional form with its value. The objective was to study the spatial variability and the correlation between maize yield (GP) and soil mechanical resistance to penetration (PR) of an Oxissol soil under conventional tillage, located at the Experimental Station of the Engineering College - FE/UNESP Ilha Solteira, São Paulo State, Brazil. It was defined 133 points of sampling in the field, according to a grid with regular intervals of $10.0 \mathrm{~m}$, for the attainment of PR, gravimetric moisture (GM), in the layers of $0-0.10 ; 0.10-0.20 ; 0.20-0.30$ and $0.30-0.40 \mathrm{~m}$, and the GP. The exponential model showed adequate to describe all the adjusted experimental semivariograms. The PR and GM showed moderate spatial dependence, with range varying between 20.2 to $63.0 \mathrm{~m}$. The GP presented strong spatial dependence with range of $15.3 \mathrm{~m}$. There was not a spatial correlation between PR and GP. Values of PR varying between 0.9 and 2.0 MPa did not limit the GP.
\end{abstract}

KEYWORDS: geostatistic, kriging, Zea mays.

\footnotetext{
${ }^{1}$ Doutorando do Curso de Pós-Graduação em Produção Vegetal, FCAV/UNESP, Departamento de Solos e Adubos, Via de Acesso Prof. Paulo Donato Castellane, s/no Jaboticabal - SP, onafreddi@ fcav.unesp.br

${ }^{2}$ Professor Livre-Docente, Departamento de Fitossanidade, Engenharia Rural e Solos, Faculdade de Engenharia, UNESP, Av. Brasil Centro 56, Caixa Postal 31, Ilha Solteira - SP, morel@agr.feis.unesp.br

${ }^{3}$ Engenheiro Agrônomo, FE/UNESP, Ilha Solteira - SP.

Recebido pelo Conselho Editorial em: 29-10-2004

Aprovado pelo Conselho Editorial em: 30-3-2006
} 


\section{INTRODUÇÃO}

A habilidade das plantas em explorar o solo, em busca de água e nutrientes, depende grandemente da distribuição de raízes no perfil desse solo que, por sua vez, depende das condições físicas e químicas, as quais são passíveis de alterações em função do manejo aplicado (ALVARENGA \& CRUZ, 2003). Os diferentes sistemas de manejo dos solos têm a finalidade de criar condições favoráveis ao desenvolvimento das culturas; entretanto, o problema de compactação/adensamento do solo vem aparecendo sistematicamente na região do Cerrado, onde os sistemas convencionais de manejo do solo promovem a desagregação excessiva da camada arável, o encrostamento superficial e a formação de camadas coesas ou compactadas, denominadas pé-de-grade ou pé-de-arado (FREITAS 1994; TAVARES FILHO et al., 2001).

A compactação é uma alteração estrutural que promove reorganização das partículas e de seus agregados (STONE et al., 2002), podendo limitar a adsorção, absorção de nutrientes, infiltração e redistribuição de água, trocas gasosas e o desenvolvimento do sistema radicular e da parte aérea (GHOHMANN \& QUEIROZ NETO, 1966), resultando em decréscimo da produtividade das culturas e aumento da energia necessária para o preparo do solo (SOANE \& OUWERKERK, 1994). Um dos atributos físicos mais adotados como indicativo da compactação do solo tem sido a resistência do solo à penetração (STONE et al., 2002), por apresentar relações diretas com o crescimento das plantas (HOAD et al., 2001) e por ser mais eficiente na identificação de estados de compactação comparada à densidade do solo (STRECK et al., 2004).

A análise da variabilidade do solo, por meio da geoestatística, pode indicar alternativas de manejo, não só para reduzir os efeitos da variabilidade do solo sobre a produção das culturas (TRANGMAR et al., 1985), mas também para aumentar a possibilidade de estimar respostas das culturas sob determinadas práticas de manejo (OVALLES \& REY, 1994). Avanços tecnológicos na agropecuária têm mostrado a importância de se conhecer a variação espacial e temporal de propriedades que afetam a produtividade das culturas, com o objetivo de otimizar o aproveitamento de recursos e diminuir custos (CARVALHO et al., 2002). Portanto, o objetivo do trabalho foi avaliar a variabilidade espacial e a correlação da resistência mecânica do solo à penetração com a produtividade de grãos da cultura do milho.

\section{MATERIAL E MÉTODOS}

O trabalho foi desenvolvido na Fazenda de Ensino e Pesquisa da Faculdade de Engenharia de Ilha Solteira (FE/UNESP), localizada no município de Selvíria (MS), latitude $20^{\circ} 18^{\prime} 05^{\prime \prime}$ 'S e longitude $52^{\circ} 40^{\prime} 28^{\prime \prime} \mathrm{W}$. A precipitação e a temperatura média anual são de $1.300 \mathrm{~mm}$ e $23,7^{\circ} \mathrm{C}$, respectivamente. O tipo climático é Aw, segundo Köeppen, caracterizado como tropical úmido com estação chuvosa no verão e seca no inverno. O estudo foi realizado em um Latossolo Vermelho distrófico típico, textura argilosa, A moderado, hipodistrófico, álico, caulinítico, mesoférrico, mesodênsico, mesocompactado, muito profundo, ácido (EMBRAPA, 1999).

Foi utilizado o preparo convencional do solo para a implantação da cultura do milho, realizandose uma aração com arado de aiveca na profundidade de $0,30 \mathrm{~m}$, uma gradagem pesada seguida de uma niveladora, respectivamente, nas profundidades de $0,15 \mathrm{~m} \mathrm{e} 0,10 \mathrm{~m}$. $\mathrm{O}$ solo foi analisado quimicamente, segundo metodologia citada por RAIJ et al. (1987), e adubado com $220 \mathrm{~kg} \mathrm{ha}^{-1} \mathrm{da}$ fórmula 08-28-16 para a obtenção da produtividade de grãos esperada de 6 a $8 \mathrm{t} \mathrm{ha}^{-1}$, segundo RAIJ et al. (1997). A semeadura do milho foi realizada em 18-11-2002, utilizando-se da variedade SEMEALI XB 8010, com distribuição de 5,4 sementes por metro. Para a adubação de cobertura, utilizou-se de $150 \mathrm{~kg}$ de uréia, aos 47 dias após a semeadura. 
Para a avaliação da variabilidade espacial, foi montada uma malha retangular no espaçamento de 10 x 10 m estabelecida entre dois terraços, utilizando-se, para isso, de um nível ótico comum, totalizando 66 pontos. Foi realizada também a adição de 67 pontos, espaçados de 1 x $1 \mathrm{~m}$, visando a detalhar o estudo da dependência espacial dos dados para condições menores do que $10 \mathrm{~m}$, totalizando 133 pontos.

Os atributos do solo determinados foram a resistência mecânica do solo à penetração (RP) e a umidade gravimétrica (UG). O parâmetro da planta avaliado foi a produtividade de grãos (PG).

Em dezembro de 2002, foram determinados os valores da variável resistência mecânica do solo à penetração (RP) nas camadas de 0-0,10 $\left(\mathrm{RP}_{1}\right)$; 0,10-0,20 $\left(\mathrm{RP}_{2}\right)$; 0,20-0,30 $\left(\mathrm{RP}_{3}\right)$ e 0,30-0,40 $\mathrm{m}\left(\mathrm{RP}_{4}\right)$ com penetrômetro de impacto (STOLF, 1991) e os valores da umidade gravimétrica (UG), segundo EMBRAPA (1979), obtidos por meio de trado de rosca, nas mesmas camadas, em todos os pontos da malha. Assim, ficaram representadas por $\mathrm{UG}_{1}, \mathrm{UG}_{2}, \mathrm{UG}_{3}$ e $\mathrm{UG}_{4}$, da superfície para a subsuperfície do solo. A expressão utilizada para o cálculo da RP foi:

$$
\mathrm{RP}=\{5,6+6,89[\mathrm{~N} /(\mathrm{P}-\mathrm{A}) 10]\} 0,0981
$$

em que,

$\mathrm{RP}$ - resistência mecânica do solo à penetração, em MPa;

$\mathrm{N}$ - número de impactos efetuados com o martelo para a obtenção da leitura, e

A e P - leituras antes e após a realização dos impactos, $\mathrm{cm}$.

A PG foi obtida pela coleta das plantas no entorno de cada ponto da malha. A área útil para

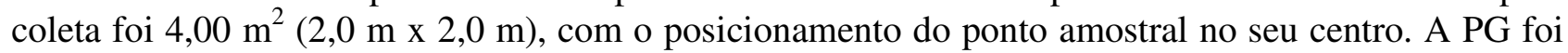
representada pela massa de grãos das espigas contidas na área de coleta corrigida para 13\% de umidade e transformada para a área de um hectare.

Os resultados foram submetidos aos procedimentos da estatística descritiva, para a obtenção da média, mediana, desvio-padrão, coeficientes de variação, assimetria e curtose. Também foi efetuada a análise de distribuição de freqüência dos dados, visando-se a testar a hipótese de normalidade pelo teste de SHAPIRO \& WILK (1965), a $1 \%$ de probabilidade.

A análise de dependência espacial foi avaliada utilizando-se das técnicas da geoestatística, nas quais o semivariograma assume papel fundamental na estimativa da dependência espacial entre amostras. Os valores de $\mathrm{R}^{2}$ (coeficiente de determinação) e SQR (soma dos quadrados dos resíduos) foram utilizados na seleção dos modelos dos semivariogramas, descritos em VIEIRA et al. (1983). O semivariograma foi estimado por meio da seguinte equação:

$$
\hat{\gamma}(h)=\frac{1}{2 N(h)} \sum_{i=1}^{N(h)}\left[Z\left(x_{i}\right)-Z\left(x_{i}+h\right)\right]^{2}
$$

em que,

$\mathrm{N}(\mathrm{h})$ - número de pares de valores medidos $\mathrm{Z}\left(\mathrm{x}_{\mathrm{i}}\right), \mathrm{Z}\left(\mathrm{x}_{\mathrm{i}}+\mathrm{h}\right)$, separados pelo vetor $\mathrm{h}$.

O semivariograma, normalmente, é representado pelo gráfico de $\hat{y}(h)$ versus $h$, ou seja, a semivariância do atributo versus a distância (VIEIRA, 1997). Os semivariogramas forneceram estimativas dos parâmetros efeito pepita $\left(\mathrm{C}_{0}\right)$, patamar $\left(\mathrm{C}_{0}+\mathrm{C}\right)$ e alcance $(\mathrm{a})$. $\mathrm{O}$ efeito pepita $\left(\mathrm{C}_{0}\right)$ é o parâmetro do semivariograma que indica a variabilidade não explicada pelos modelos ajustados, considerando a distância de amostragem utilizada (CAMBARDELLA et al., 1994). O efeito pepita puro é o valor da semivariância para distância zero e representa o componente da variação ao acaso. $\mathrm{O}$ patamar $\left(\mathrm{C}_{0}+\mathrm{C}\right)$ é o valor da semivariância em que a curva estabiliza sobre um valor constante (TRANGMAR et al., 1985), ou seja, à medida que $\mathrm{h}$ aumenta, $\hat{\mathrm{y}}(\mathrm{h})$ também aumenta até um valor máximo no qual se estabiliza. $\mathrm{O}$ alcance (a) indica que amostras localizadas a distâncias menores que o 
alcance são dependentes espacialmente umas das outras. $\mathrm{O}$ alcance depende do tamanho da área amostrada e da escala de observação, sendo tanto maior quanto maior o intervalo entre medidas (TRANGMAR et al., 1985). A análise da dependência espacial (ADE) foi efetuada segundo a expressão contida em ROBERTSON (1998):

$$
\mathrm{ADE}=[\mathrm{C} /(\mathrm{C}+\mathrm{Co})] 100
$$

em que,

ADE - estimador da dependência espacial (\%);

C - variância estrutural, e

$\mathrm{C}+\mathrm{C}_{0}$ - patamar.

Foram testados os modelos esférico, exponencial e gaussiano, sendo que todos os dados se ajustaram ao modelo exponencial $(\operatorname{Exp}), \hat{\mathrm{y}}(\mathrm{h})=\mathrm{C}_{0}+\mathrm{C}[1-\exp (-3 \mathrm{~h} / \mathrm{a})]$ para $0<\mathrm{h}<\mathrm{d}$, em que $\mathrm{d}$ é a distância máxima na qual o semivariograma é definido.

Em seguida à modelagem dos semivariogramas, foi realizada a interpolação por krigagem, sendo essa uma técnica de interpolação para estimativa de valores de uma propriedade em locais nãoamostrados, a partir de 16 vizinhos amostrados na malha. A krigagem, no entanto, faz uso de um interpolador linear não-tendencioso e de variância mínima, que assegura a melhor estimativa dos dados não-amostrados. Esse estimador tem como base os dados amostrais da variável regionalizada e as propriedades estruturais do semivariograma obtido a partir desses dados (VIEIRA et al., 1983). Testou-se a obtenção do semivariograma cruzado, entre a RP e a PG, que é uma ferramenta geoestatística que permite verificar o relacionamento entre as variabilidades de duas séries de valores espacialmente medidos, tornando possível avaliar se a variabilidade de uma série é acompanhada pela da outra, de forma direta como indireta (SOUZA, 1992). Também foi efetuado o estudo de regressão linear entre a RP e a PG.

\section{RESULTADOS E DISCUSSÃO}

O conhecimento da distribuição de frequiência dos dados de uma variável tem importantes conseqüências práticas, uma vez que os métodos de análise a serem aplicados são distintos para as diferentes distribuições. Exemplificando, sabe-se que a média é uma boa medida descritiva para dados com distribuição normal. No entanto, o mesmo não acontece para aqueles com distribuição lognormal, para os quais a mediana é mais adequada como medida de posição que a média (SPIEGEL, 1985). Assim, a análise da Tabela 1 revelou que todos os parâmetros estudados possuíram distribuição de freqüência do tipo normal, sendo a média uma boa medida para compará-los, exceção feita apenas para a $\mathrm{RP}_{1} \mathrm{e} \mathrm{RP}_{4}$. Entretanto, a normalidade dos dados não é exigência da geoestatística, sendo conveniente apenas que a distribuição não apresente caudas muito alongadas, o que poderia comprometer as análises (WEBSTER, 1985).

Segundo PAZ-GONZALES et al. (2001), a estimação por krigagem apresenta melhores resultados quando a normalidade dos dados é satisfeita, e, ainda, segundo GONÇALVES et al. (2001), a distribuição normal dos dados é muito interessante na avaliação da dependência espacial, porém mais importante que a normalidade é a verificação de que os dados não apresentem tendência, o que não foi observado.

Para avaliar a variabilidade dos dados, analisaram-se os coeficientes de variação, conforme o critério de classificação proposto por GOMES (2000): baixo (C.V. < 10\%); médio $(10 \%<$ C.V. $<$ $20 \%$ ); alto $(20 \%<$ C.V. < $30 \%)$ e muito alto (C.V. $>30 \%)$, o qual tem sido adotado em muitos trabalhos relacionados com estudos de solo, por ser uma medida adimensional e possibilitar a comparação da variabilidade de duas variáveis. 
TABELA 1. Análise descritiva da produtividade de grãos (PG) da cultura do milho, da umidade gravimétrica (UG) e de resistência mecânica à penetração (RP) de um Latossolo Vermelho.

\begin{tabular}{lccccccc}
\hline \multirow{2}{*}{ Atributo } & Média & Mediana & Variância & \multicolumn{3}{c}{ Coeficiente } & \multicolumn{2}{c}{\begin{tabular}{c} 
Distribuição \\
\cline { 5 - 7 }
\end{tabular}} & & & & Variação $(\%)$ & Curtose & Assimetria $\begin{array}{c}\text { de } \\
\text { freqüência }\end{array}$ \\
\hline $\mathrm{UG}_{1}\left(\mathrm{~kg} \mathrm{~kg}^{-1}\right)$ & 0,163 & 0,163 & $2,76910^{-4}$ & 10,2 & $-0,174$ & 0,085 & $\mathrm{~N}$ \\
$\mathrm{UG}_{2}\left(\mathrm{~kg} \mathrm{~kg}^{-1}\right)$ & 0,188 & 0,188 & $5,71210^{-5}$ & 4,0 & $-0,617$ & 0,203 & $\mathrm{~N}$ \\
$\mathrm{UG}_{3}\left(\mathrm{~kg} \mathrm{~kg}^{-1}\right)$ & 0,196 & 0,195 & $7,68010^{-5}$ & 4,5 & $-0,068$ & 0,284 & $\mathrm{~N}$ \\
$\mathrm{UG}_{4}\left(\mathrm{~kg} \mathrm{~kg}^{-1}\right)$ & 0,207 & 0,207 & $9,94310^{-5}$ & 4,8 & $-0,598$ & $-0,043$ & $\mathrm{~N}$ \\
$\mathrm{RP}_{1}(\mathrm{MPa})$ & 0,933 & 0,752 & $1,91210^{-1}$ & 46,9 & $-0,375$ & 0,859 & $\mathrm{~L}$ \\
$\mathrm{RP}_{2}(\mathrm{MPa})$ & 1,501 & 1,543 & $2,18610^{-1}$ & 31,2 & $-0,529$ & $-0,090$ & $\mathrm{~N}$ \\
$\mathrm{RP}_{3}(\mathrm{MPa})$ & 2,036 & 2,078 & $1,02910^{-1}$ & 15,8 & $-0,077$ & $-0,366$ & $\mathrm{~N}$ \\
$\mathrm{RP}_{4}(\mathrm{MPa})$ & 2,067 & 2,014 & $9,15010^{-2}$ & 14,6 & 0,015 & 0,489 & $\mathrm{~L}$ \\
$\left.\mathrm{PG}_{(\mathrm{t} \mathrm{ha}}{ }^{-1}\right)$ & 6,024 & 6,003 & 2,886 & 28,2 & $-0,570$ & 0,210 & $\mathrm{~N}$ \\
\hline
\end{tabular}

(a) $\mathrm{N}$ - distribuição normal; L - distribuição lognormal

A variabilidade da UG foi classificada como média para $\mathrm{UG}_{1}$ e baixa nas demais. Para RP foi alta na $\mathrm{RP}_{1}$ e $\mathrm{RP}_{2}$ e média para as demais. Observou-se diminuição da variabilidade desses atributos com o aumento da profundidade do solo. Provavelmente, a maior variabilidade encontrada nas camadas superficiais do solo seja proporcionada pelos implementos utilizados em seu preparo, principalmente pelas grades na camada de $0-0,20 \mathrm{~m}$. Para o atributo da planta, tal variabilidade ficou classificada como alta para a PG, corroborando o observado por TRANGMAR et al. (1987), que relataram haver menor variabilidade dos atributos do solo em relação aos parâmetros da planta. Notouse que, em todas as camadas, os valores de UG estavam próximos da capacidade de campo $\left(0,20 \mathrm{~kg} \mathrm{~kg}^{-1}\right)$, determinada nesse Latossolo por MARTINS (2002), não interferindo na análise da RP que é inversamente influenciada pela umidade do solo, com exceção apenas para $\mathrm{RP}_{1}$, que apresentou, mesmo assim, valores de RP abaixo de $1 \mathrm{MPa}$ (Tabela1).

Observa-se, pelas equações de regressão contidas na Tabela 2, que a RP, na camada de 0-0,30 m, não restringiu a PG, o que pôde ser verificado para camada de 0,30-0,40 m, possivelmente por não ter sofrido influências do preparo do solo.

TABELA 2. Regressão linear entre a produtividade de grãos (PG) em função da resistência mecânica à penetração (RP) de um Latossolo Vermelho.

\begin{tabular}{ccc}
\hline Camada $(\mathrm{m})$ & Equação & $\mathrm{R}^{2}$ \\
\hline $0-0,10$ & $\mathrm{PG}=6,135+1,495 \mathrm{RP}_{1}$ & $0,170^{*}$ \\
$0,10-0,20$ & $\mathrm{PG}=1,283+0,036 \mathrm{RP}_{2}$ & $0,131^{*}$ \\
$0,20-0,30$ & $\mathrm{PG}=4,124+0,933 \mathrm{RP}_{3}$ & $0,176^{*}$ \\
$0,30-0,40$ & $\mathrm{PG}=7,451-4,590 \mathrm{RP}_{4}$ & $-0,170^{*}$ \\
\hline
\end{tabular}

* significativo a $5 \%$ de probabilidade; ${ }^{\text {ns }}$ não-significativo.

Portanto, o sinal positivo do b na camada de $0-0,30 \mathrm{~m}$, além de ter indicado a desestruturação do solo proporcionado pelo preparo convencional, sugeriu a necessidade de leve compactação dessa camada com o objetivo de aumentar o contato solo/raiz e, por conseguinte, aumentar a PG. Segundo HAKANSSON et al. (1998), solos excessivamente soltos apresentam menores produtividades por causa do menor contato solo/raiz, diminuindo a habilidade do sistema radicular em absorver quantidades adequadas de água e nutrientes. Em contrapartida, o sinal negativo do b $(0,30-0,40 \mathrm{~m})$ sugeriu a necessidade de descompactação dessa camada, com o objetivo de aumentar a respiração 
celular das raízes e, conseqüentemente, também proporcionar aumento da PG. Contudo, os coeficientes de correlação, observados para as regressões lineares estabelecidas entre a PG e as RPs, não ultrapassaram 17,6\%. Portanto, esse resultado evidenciou que, no máximo, apenas 17,6\% da variação da PG puderam ser explicados pela variação da RP, ficando o restante por conta de outros fatores.

A análise geoestatística, efetuada por meio do semivariograma, demonstrou que os atributos $\mathrm{UG}_{1}, \mathrm{UG}_{3}, \mathrm{RP}_{4}$ e $\mathrm{PG}$ apresentaram dependência espacial e ajustaram-se ao modelo exponencial (Tabela 3). Em contrapartida, a $\mathrm{UG}_{2}, \mathrm{UG}_{4}, \mathrm{RP}_{1}, \mathrm{RP}_{2}$ e $\mathrm{RP}_{3}$ resultaram efeito pepita puro, não havendo dependência espacial nesses atributos, o que significa, possivelmente, que o alcance para os dados em questão é menor do que o menor espaçamento entre amostras, apresentando distribuição espacial aleatória (Tabela 1).

TABELA 3. Parâmetros dos semivariogramas ajustados à produtividade de grãos (PG) da cultura do milho e à resistência mecânica à penetração (RP) de um Latossolo Vermelho.

\begin{tabular}{lccccccc}
\hline \multirow{2}{*}{ Atributo } & \multicolumn{7}{c}{ Parâmetros do Semivariograma } \\
\cline { 2 - 8 } & Modelo $^{(\mathrm{a})}$ & $\begin{array}{c}\text { Efeito Pepita } \\
(\mathrm{Co})\end{array}$ & $\begin{array}{c}\text { Patamar } \\
(\mathrm{Co}+\mathrm{C})\end{array}$ & $\begin{array}{c}\text { Alcance } \\
(\mathrm{m})\end{array}$ & $\mathrm{R}^{2}$ & $\mathrm{SQR}^{(\mathrm{b})}$ & $\mathrm{ADE}^{(\mathrm{c})}$ \\
\hline $\mathrm{UG}_{1}\left(\mathrm{~kg} \mathrm{~kg}^{-1}\right)$ & exponencial & $1,75010^{-4}$ & $3,06010^{-4}$ & 41,5 & 0,636 & $6,35910^{-1}$ & 42,9 \\
$\mathrm{UG}_{2}\left(\mathrm{~kg} \mathrm{~kg}^{-1}\right)$ & epp & $4,64210^{-5}$ & --- & --- & --- & --- & --- \\
$\mathrm{UG}_{3}\left(\mathrm{~kg} \mathrm{~kg}^{-1}\right)$ & exponencial & $1,82410^{-5}$ & $5,08810^{-5}$ & 20,2 & 0,941 & $9,41110^{-1}$ & 73,6 \\
$\mathrm{UG}_{4}\left(\mathrm{~kg} \mathrm{~kg}^{-1}\right)$ & epp & $5,39710^{-5}$ & --- & --- & --- & --- & --- \\
$\mathrm{RP}_{1}(\mathrm{MPa})$ & epp & $1,94210^{-1}$ & --- & --- & --- & --- & --- \\
$\mathrm{RP}_{2}(\mathrm{MPa})$ & epp & $2,14310^{-1}$ & --- & --- & --- & --- & --- \\
$\mathrm{RP}_{3}(\mathrm{MPa})$ & epp & $9,70710^{-2}$ & --- & --- & --- & --- & --- \\
$\mathrm{RP}_{4}(\mathrm{MPa})$ & exponencial & $1,06210^{-2}$ & $2,37610^{-2}$ & 63,0 & 0,818 & $8,18010^{-1}$ & 55,3 \\
$\left.\mathrm{PG}_{(\mathrm{t} \mathrm{ha}}{ }^{-1}\right)$ & exponencial & $5,23710^{-1}$ & 2,919 & 15,3 & 0,598 & $5,98410^{-1}$ & 82,1 \\
\hline
\end{tabular}

(a) epp - efeito pepita puro; (b) SQR - soma dos quadrados dos resíduos; (c) ADE - avaliador da dependência espacial

A constatação da dependência espacial para $\mathrm{UG}_{1}, \mathrm{UG}_{3}, \mathrm{RP}_{4}$ e $\mathrm{PG}$ evidenciou que a distribuição de tais atributos no espaço não é aleatória, uma vez que suas classes de dependência espacial, determinadas pelo ADE, variaram da seguinte forma: a) UG: de 42,9 a 73,6\% (moderada); b) RP: 55,3\% (moderada), e c) atributo da planta: 82,1\% (forte). Portanto, em função das classes de dependência espacial obtidas para $\mathrm{RP}_{4}$ e a PG, pôde-se constatar que 82,1\% da variação total da PG foi explicada pela dependência espacial, ao qual, por outro lado, ficou atribuído erro ao acaso, referente ao efeito pepita, de $17,9 \%$. De forma semelhante para a $\mathrm{RP}_{4}$, tais cifras foram, respectivamente, de $55,3 \%$ e de $44,7 \%$. Os alcances da dependência espacial variaram entre 20,2 e $63,0 \mathrm{~m}$ para os atributos do solo, e foi de $15,3 \mathrm{~m}$ para a PG.

Valores de resistência mecânica à penetração, variando de 2,0 a 4,0 MPa, segundo ARSHAD et al. (1996), podem restringir ou mesmo impedir o crescimento e o desenvolvimento das raízes. Entretanto, TAVARES FILHO et al. (2001) demonstraram que valores de resistência superiores a 3,5 MPa não restringiram o desenvolvimento radicular e a produtividade de grãos do milho, influenciando apenas em sua morfologia. Para o conceito de intervalo hídrico ótimo, SILVA et al. (1994) relacionaram o valor médio de 2,0 MPa como limite para o desenvolvimento radicular. Os valores médios da RP observados para o Latossolo Vermelho variaram entre 0,933 e 1,501 na camada de 0-0,20 m, e 2,024 e 2,067 MPa na camada de 0,20-0,40 m, ficando abaixo dos valores críticos de 
RP para o desenvolvimento e produtividade de grãos da cultura do milho encontrados na literatura, o que pôde ser comprovado pela produtividade média atingida de 6,024 $\mathrm{t} \mathrm{ha}^{-1}$ (Tabela 1).

Para visualizar a distribuição dos valores de $\mathrm{RP}_{4}$ e $\mathrm{PG}$, realizou-se a krigagem dos dados, gerando mapas de superfície (Figura 1). Observa-se, pelos mapas de krigagem, que não houve correlação espacial entre a variabilidade da $\mathrm{PG}$ em função da $\mathrm{RP}_{4}$, o que pôde ser confirmado pela falta de estrutura do semivariograma cruzado entre os dois atributos. Verifica-se, também, que $82 \%$ da área mapeada apresentou resistência mecânica à penetração entre 1,8 e 2,05 MP na camada de $0,30-0,40 \mathrm{~m}$, e que $83 \%$ da área apresentou produtividade acima de 5,6 $\mathrm{t} \mathrm{ha}^{-1}$, evidenciando claramente o predomínio de elevadas produtividades da cultura e a não-interferência da $\mathrm{RP}_{4}$ sobre a $\mathrm{PG}$. Portanto, uma das vantagens dos mapas de krigagem é a exata localização de áreas com baixas produtividades, o que seria impossível somente com os dados médios (Tabela 1).
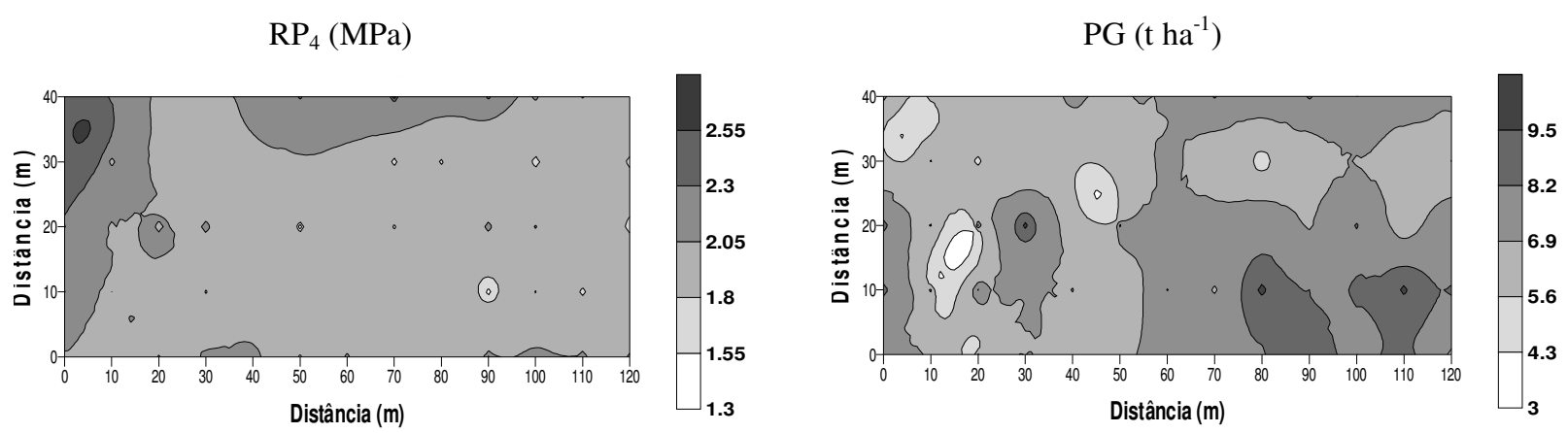

FIGURA 1. Mapas de isolinhas da resistência mecânica à penetração (RP) e da produtividade de grãos (PG) da cultura do milho cultivado sob Latossolo Vermelho.

\section{CONCLUSÕES}

A resistência mecânica do Latossolo Vermelho à penetração, submetido ao preparo convencional, apresentou moderada dependência espacial apenas na camada de $0,30-0,40 \mathrm{~m}$, com alcance de 63,0 m. Não houve correlação espacial entre a resistência mecânica do solo à penetração e a produtividade de grãos do milho. Valores de resistência mecânica à penetração variando entre $0,9 \mathrm{e}$ 2,0 MPa não restringiram a produtividade da cultura do milho.

\section{REFERÊNCIAS}

ARSHAD, M.A.; LOWERY, B.; GROSSMAN, B. Physical tests for monitoring soil quality. In: DORAN, J.W.; JONES, A.J. Methods for assessing soil quality. Madison: Soil Science Society of America, 1996. p.123-41.

ALVARENGA, R.C.; CRUZ, J.C. Manejo de solos e agricultura irrigada. In: RESENDE, M.; ALBUQUERQUE, P.E.P.; COUTO, L. A cultura do milho irrigado. Brasília: Embrapa Informação Tecnológica, 2003. p.70-106.

CAMBARDELLA, C.A.; MOORMAN, T.B.; NOVAK, J.M.; PARKIN, T.B.; KARLEN, D.L.; TURCO, R.F.; KONOPKA, A.E. Field-scale variability of soil properties in Central Iowa Soils. Soil Science Society America Journal, Madison, v.58, p.1501-11, 1994.

CARVALHO, J.R.; SILVEIRA, P.M.; VIEIRA, S.R. Geoestatística na determinação da variabilidade espacial de características químicas do solo sob diferentes preparos. Pesquisa Agropecuária Brasileira, Brasília, v.37, n.8, p.1151-9, 2002. 
EMPRESA BRASILEIRA DE PESQUISA AGROPECUÁRIA - EMBRAPA. Centro Nacional de pesquisa de Solos. Sistema brasileiro de classificação de solos. Rio de Janeiro, 1999. 412 p.

EMPRESA BRASILEIRA DE PESQUISA AGROPECUÁRIA - EMBRAPA. Serviço Nacional de Levantamento e Conservação de Solos. Manual de métodos de análise de solo. Rio de Janeiro, 1979.

FREITAS, P.L. Aspectos físicos e biológicos do solo. In: LANDERS, J.N. Fascículos sobre experiências em plantio direto nos Cerrados. Uberlândia: APDC, 1994. p.187-96.

GHOHMANN, F.; QUEIROZ NETO, J.P. Efeito da compactação artificial de dois solos limoargilosos sobre a penetração de raízes de arroz. Bragantia, Campinas, v.25, n.39, p.421-31, 1966.

GOMES, P.F. Curso de estatística experimental. 14. ed. Piracicaba: Degaspari, 2000. 477 p.

GONÇALVES, A.C.A.; FOLEGATTI, M.V.; MATA, J.D.V. Análises exploratória e geoestatística da variabilidade de propriedades físicas de um Argissolo Vermelho. Acta Scientiarum, Maringá, v.23, n.5, p.1149-57, 2001.

HAKANSSON, I.; STENBERG, M.; RYDBERG, T. Long term experiments with different depths of mouldboard plough in Sweden. Soil and Tillage Research, Amsterdam, v.46, n.3-4, p.209-23, 1998.

HOAD, S.P.; SILVA, A.P.; DIAS JUNIOR, M.S.; TORMENA, C.A. Quantificação de pressões críticas para o crescimento das plantas. Revista Brasileira de Ciência do Solo, Viçosa, v.25, n.1 , p.11$18,2001$.

MARTINS, C.B. Análise da resistência mecânica à penetração de um Latossolo Vermelho distrófico em função do grau de umidade. 2002. 46 f. Monografia (Trabalho de Graduação) - Faculdade de Engenharia de Ilha Solteira, Universidade Estadual Paulista, Ilha Solteira, 2002.

OVALLES, F.; REY, J. Variabilidad interna de unidades de fertilidad en suelos de la depresión del Lago de Valencia. Agronomia Tropical, Maracay, v.44, n.1, p.41-65, 1994.

PAZ-GONZALEZ, A.; TABOADA CASTRO, M.T.; VIEIRA, S.R. Geostatistical analysis of heavy metals in a one-hectare plot under natural vegetation in a serpentine area. Canadian Journal of Soil Science, Ottawa, v.81, n.2, p.469-79, 2001.

RAIJ, B. van; CANTARELLA, H.; QUAGGIO, J.A.; FURLANI, A.M.C. Recomendações de adubação e calagem para o estado de São Paulo. Campinas: Instituto Agronômico/Fundação IAC, 1997. $285 \mathrm{p}$.

RAIJ, B. van; QUAGGIO, J.A.; CANTARELLA, H.; FERREIRA, M.; LOPES, A.S.; BATAGLIA, O.C. Análise química do solo para fins de fertilidade. Campinas: Fundação Cargill, 1987. 170 p.

ROBERTSON, G.P. GS+: geostatistics for the enviromental sciences. Plainwell: Michigan, 1998. 152 p.

SILVA, A.P.; KAY, B.D.; PERFECT, E. Characterization of the least limiting water range. Soil Science Society of America Journal, Madison, v.58, p.1775-81, 1994.

SPIEGEL, M.R. Estatística. 2.ed. São Paulo: McGraw-Hill, 1977. 454 p.

SHAPIRO, S.S.; WILK, M.B. An analysis of variance test for normality: complete samples. Biometrika, London, v.52, n.2, p.591-611, 1965.

SOANE, B.D.; OUWERKERK, C. Soil compaction in crop production. Amsterdam: Elsevier, 1994. $660 \mathrm{p}$. 
SOUZA, L.S. Variabilidade espacial do solo em sistemas de manejo. $1992.162 \mathrm{f}$. Tese (Doutorado em Ciência do Solo) - Faculdade de Agronomia, Universidade Federal do Rio Grande do Sul, Porto Alegre, 1992.

STONE, L.F.; GUIMARÃES, C.M.; MOREIRA, J.A.A. Compactação do solo na cultura do feijoeiro 1: efeitos nas propriedades físico-hídricas do solo. Revista Brasileira de Engenharia Agrícola e Ambiental, Campina Grande, v.6, n.2, p.207-12, 2002.

STOLF, R.Teoria e teste experimental de fórmulas de transformação dos dados de penetrômetro de impacto em resistência do solo. Revista Brasileira de Ciência do Solo, Viçosa, v.15, n.3, p.229-35, 1991.

STRECK, C.A.; REINERT, D.J.; REICHERT, J.M.; KAISER, D.R. Modificações em propriedades físicas com a compactação do solo causada pelo tráfego induzido de um trator em plantio direto. Ciência Rural, Santa Maria, v.34, n.3, p.755-60, 2004.

TAVARES FILHO, J.; BARBOSA, G.M.C.; GUIMARÃES, M.F.; FONSECA, I.C.B. Resistência do solo à penetração e desenvolvimento do sistema radicular do milho (Zea mays, L.) sob diferentes sistemas de manejo em um latossolo roxo. Revista Brasileira de Ciência do Solo, Viçosa, v.25, n.3, p.725 -30, 2001.

TRANGMAR, B.B.; YOST, R.S.; UEHARA, G. Application of geostatistics to spatial studies of soil properties. Advances in Agronomy, San Diego, v.38, n.1 p.45-94, 1985.

TRANGMAR, B.B.; YOST, R.S.; WADE, M.K.; UEHARA, G.; SUDJADI, M. Spatial variation of soil properties and rice yield on recently cleared land. Soil Science Society America Journal, Madison, v.51, n.3, p.668-74, 1987.

VIEIRA, S.R.; HATFIELD, J.L.; NIELSEN, D.R.; BIGGAR, J.W. Geostatistical theory and application to variability of some agronomical properties. Hilgardia, Berkeley, v.51, n.3, p.1-75, 1983.

VIEIRA, S.R.; TILLOTSON, P.M.; BIGGAR, J.W.; NIELSEN, D.R. Scaling of semivariograms and the kriging estimation of field-measured properties. Revista Brasileira de Ciência do Solo, Viçosa, v.21, n.3, p.525-33, 1997.

WEBSTER, R. Quantitative spatial analyses of soil in field. New York: Springer-Verlag, 1985. 56 p. 